Ann. Zootech., I966, 15 (3), 29I-295.

\title{
ÉTUDE COMPARATIVE DE DIFFÉRENTES MÉTHODES DE MESURE DU POUVOIR DE RÉTENTION D'EAU DE LA VIANDE DE PORC
}

\author{
R. GOUTEFONGEA \\ Laboratoire de Recherches sur la Viande, \\ Centre national de Recherches zootechniques, 78 - Jouy-en-Josas
}

\section{SOMMAIRE}

Six méthodes de détermination du pouvoir de rétention d'eau de la viande de porc ont été comparées. Elles faisaient appel à la pression ou à la centrifugation, cette dernière avec ou sans addition d'eau.

Des différences significatives entre les différentes méthodes ont été observées. La technique retenue consiste à presser un échantillon de $5 \mathrm{~g}$ de broyat entre deux feuilles de papier-filtre pendant I $\mathrm{mn}$ à l'aide d'un piston.

La myopathie exsudative et dépigmentaire du porc se caractérise post mortem par des modifications de certaines caractéristiques physico-chimiques du muscle, en particulier par un pouvoir de rétention d'eau faible comparativement aux muscles d'animaux normaux, ce qui se traduit par un exsudat plus ou moins important à la surface des muscles.

Toute étude portant sur les viandes exsudatives nécessite donc de disposer de méthodes de mesure du pouvoir de rétention d'eau musculaire. La mesure de cette caractéristique permet, outre l'appréciation objective de l'affection, de prévoir, au moins en partie, l'évolution tultérieure de la viande, ce qui fournit des informations intéressantes en vue de son utilisation et de sa commercialisation (pertes en chambre froide, à la décongélation, à la cuisson, facilité de conservation, rendement technologique, etc.).

Le pouvoir de rétention d'eau du muscle traduit la force de la liaison de l'eau aux protéines musculaires. Toutefois, compte tenu des différents types de liaison de l'eau à ces protéines, on ne peut guère concevoir de mesure du pouvoir de rétention d'eau en valeur absolue; en effet, des échantillons musculaires soumis à des conditions d'extraction précises perdent une partie de l'eau qu'ils contiennent, qui est ainsi définie comme " eau libre ", l'eau restante étant appelée, par différence, "l'eau liée ", mais il est bien évident que chaque méthode d'extraction définit une eatı " liée " et une eau " libre " et que l'on ne peut comparer le pouvoir de rétention d'eau de différents échantillons que s'ils ont été soumis aux mêmes conditions.

Diverses méthodes ont été proposées pour l'extraction de l'eau " libre " de la 
viande. Nous en avons donné une description succincte (GouTfFonGEA, I963 $a$, Ig63 b). Ces méthodes font appel, dans leur quasi-totalité, à la pression ou à la centrifugation. Au cours de travaux antérieurs, nous avons été amenés à utiliser plusieurs de ces méthodes de mesure du pouvoir de rétention d'eau reposant sur les principes suivants :

\section{Pression \\ Centrifugation avec addition d'eau \\ Centrifugation à sec sur verre fritté.}

Nous avons observé qu'il existe d'importantes différences de sensibilité et de fidélité entre ces techniques lorsque l'on fait varier l'intensité de la pression, la vitesse de centrifugation, le poids de l'échantillon et la durée.

Afin de disposer d'une méthode satisfaisante, nous avons réalisé une étude comparative des techniques les plus sensibles.

\section{MATÉRIEL ET MÉTHODES}

\section{Préparation des échantillons}

Sur 4I porcs, produits d'un croisement entre un verrat de la race de Piétrain et de 6 truies de race Large White, on prélevait la partie supérieure du Long vaste 24 heures après l'abattage. Après mesure du pH, cet échantillon était broyé au moyen d'un hachoir muni d'une grille à trous de $3 \mathrm{~mm}$ de diamètre. Les déterminations étaient faites en double sur le broyat ainsi obtenu.

\section{Techniques d'extraction}

Nous avons utilisé 3 types de méthodes:

- Une méthode de pression dérivée de celle de Grau et Hamm (1952) et précédemment exposée (GoutefongeA, I960); l'échantillon est pressé entre deux feuilles de papier-filtre Whatmann 3 MM. (La pression est obtenue par un piston que l'on fait tomber d'une hauteur constante.)

- Une méthode de centrifugation avec addition d'eau au broyat (SwIFT et BERman, r959) : dans un tube de centrifugeuse de $50 \mathrm{ml}$ on homogénéise $5 \mathrm{~g}$ de broyat et $7,5 \mathrm{ml}$ d'eau à l'aide d'un microbroyeur tournant $\grave{a}_{1} 5000 \mathrm{t} / \mathrm{mn}$ pendant 3 fois 10 secondes. L'homogénat est ensuite centrifugé.

- Une méthode de centrifugation à sec sur verre fritté (Wierbicki, Kunkle, Deatherage, 1957) : le broyat est placé dans un creuset filtrant de porosité o ; ce creuset est lui-même placé, pour la centrifugation, dans un tube de $100 \mathrm{ml}$ en matière plastique.

Pour chacun de ces trois types de méthodes, nous avons fait varier les conditions d'extraction et nous avons donc comparé au total six techniques dont les caractéristiques étaient :

I.

Pression

2.

Pression

3.

4.
Poids d'échantillon .............

Poids du piston ...............

Durée de l'épreuve ...........

Poids d'échantillon.............

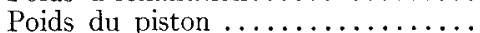

Durée de l'épreuve .............

Poids d'échantillon.............

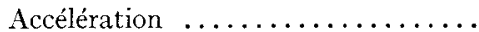

Durée

Poids de l'échantillon ............

Accélération ..............

Durée
$5 \mathrm{~g}$

$250 \mathrm{~g}$

I $\mathrm{mn}$

$5 \mathrm{~g}$

$2250 \mathrm{~g}$

I $\mathrm{mn}$

$5 \mathrm{~g}$

$4000 g$

I0 $\mathrm{mn}$

$5 \mathrm{~g}$

$4000 \mathrm{~g}$

$20 \mathrm{mn}$ 


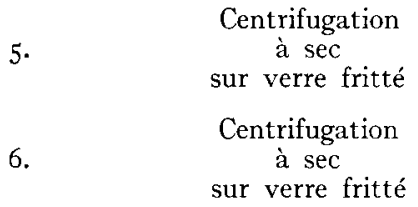

Poids d'échantillon

Accélération .................. Iо $g$

Durée...$\ldots \ldots \ldots \ldots \ldots \ldots$ Io $\mathrm{mn}$

Poids de l'échantilion ........... $3 \mathrm{~g}$

Accélération ................. $\quad 800 \mathrm{~g}$

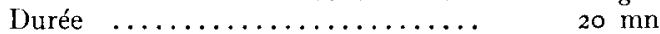

\section{Expression des résultats}

Dans tous les cas, le pouvoir de rétention d'eau était exprimé par la variation relative du poids de l'échantillon exprimée en pourcentage, soit :

$$
\frac{\text { Poids initial de l'échantillon - Poids final }}{\text { Poids initial }} \times \text { 100 }
$$

Le coefficient de fidélité (ou de répétabilité) de chaque méthode était estimé par la quantité :

$$
r=\frac{\sigma_{2} \mathrm{~S}-\sigma_{2} \mathrm{D}}{\sigma_{2} \mathrm{~S}+\sigma_{2} \mathrm{D}}
$$

$\sigma_{2} \mathrm{~S}=$ variance des sommes $\mid$ des deux valeurs obtenues par l'application de chaque méthode $\sigma_{2} \mathrm{D}=$ variance des différences $j$ à 2 échantillons du même broyat.

L'intervalle de confiance de $r$, qui est un coefficient de corrélation intra-classe, a été calculé selon la méthode donnée par FISCHER (1954).

\section{RÉSULTATS — DISCUSSION}

Le tableau I rapporte, pour chaque méthode utilisée, la moyenne des résultats, la variance et le coefficient de fidélité obtenus. Nous avons remarqué que nos échantillons présentaient une homogénéité certaine en ce qui concerne le pouvoir de rétention d'eau. Ceci est probablement dî, au moins en partie, à l'origine génétique des animaux et à l'uniformité des conditions d'élevage et du mode d'abattage. Il est donc probable que les coefficients de fidélité calculés ici sont des valeurs minimum.

TABIEAU I

\begin{tabular}{|c|c|c|c|c|}
\hline $\begin{array}{l}\text { Méthode } \\
\text { no }\end{array}$ & Moyenne & $\begin{array}{c}\text { Variance } \\
\frac{\sigma^{2} D+\sigma^{2}}{4}\end{array}$ & $\begin{array}{l}\text { Coefficient } \\
\text { de fidélité }\end{array}$ & $\begin{array}{c}\text { Intervalle de confiance } \\
\dot{a} \mathrm{P}=0,01\end{array}$ \\
\hline $\begin{array}{l}1 \\
2 \\
3 \\
4 \\
5 \\
6\end{array}$ & $\begin{array}{l}12,04 \\
28,81 \\
10,55 \\
18,41 \\
18,91 \\
26,80\end{array}$ & $\begin{array}{l}12,48 \\
19,25 \\
60,91 \\
17,56 \\
1,4,94 \\
13,46\end{array}$ & $\begin{array}{l}0,75 \\
0,87 \\
0,93 \\
0,79 \\
0,57 \\
0,63\end{array}$ & $\begin{array}{l}0,59-0,86 \\
0,76-0,93 \\
0,87-0,97 \\
0,64-0,88 \\
0,32-0,75 \\
0,40-0,79\end{array}$ \\
\hline
\end{tabular}

Moyennes, variances et coefficients de fidélité obtenus pour les différentes méthodes 
Malgré cela, des différences significatives apparaissent entre les méthodes ; on peut noter par exemple, que les deux techniques de centrifugation sur verre fritté sont peu fidèles par comparaison avec les techniques de pression et de centrifugation avec addition d'eau.

\title{
CONCLUSIONS
}

Les deux méthodes qui présentent les coefficients de fidélité les plus élevés sont les numéros 2 et 3 ; la différence entre les deux est cependant assez faible. En outre, dans le cas de méthodes destinées à être utilisées en grande série et éventuellement en des lieux ne disposant pas de matériel de laboratoire (abattoirs par exemple), nous devons tenir compte également de la rapidité et de la simplicité d'emploi. Or, la méthode $n^{0} 2$ est plus rapide et demande un matériel beaucoup plus réduit que la méthode $n^{\circ} 3$. Nous fixons donc notre choix sur la méthode $n^{0} 2$ qui consiste à presser un échantillon de $5 \mathrm{~g}$ de broyat musculaire entre deux feuilles de papierfiltre pendant I minute à l'aide d'un piston de $2250 \mathrm{~g}$.

Reçu pour publication en juin 1966.

\section{REMERCIEMENTS}

Nous remercions L. Ollivier, de la Station centrale de Génétique animale, qui nous a autorisé à prélever des échantillons sur ses animaux et aidé de ses conseils en statistique.

\author{
SUMMARY \\ COMPARATIVE STUDY OF DIFFERENT METHODS OF MEASURING \\ WATER HOLDING CAPACITY OF PIG MEAT
}

The water holding capacity of pig meat is profoundly affected by exudative and depigmenting myopathy (watery pork). Its measurement allows an objective judgement of the severity of the disorder and gives information concerning the subsequent behaviour of the meat.

After a certain number of trials a comparative study was made of several methods of measurement either by pressing or by centrifuging and varying certain factors, strength of pressure, speed of centrifuging and duration.

From calculation of coefficients of fidelity (table $r$ ) it was possible to show significant differences between methods. In addition the speed and simplicity of the different methods were considered; the resulting choice was the method in which a sample of $5 \mathrm{~g}$ ground muscle was pressed between two filter papers for I minute by a piston of $225^{\circ} \mathrm{g}$.

\section{RÉFÉRENCES BIBLIOGRAPHIQUES}

FISCIIER R. A., I954. Statistical methods for research workers. 215-221, I2th Edition. Oliver and Boyd, Edinburgh et Londres.

Govtefongea R., ig6o. Description d'un nouvel appareil pour mesurer le pouvoir de rétention d'eau de la viande. VIth Cont. Meat Res. Workers. Utrecht. 
Goutefongea R., Ig63a. Comparaison de différentes méthodes de mesure du pouvoir de rétention d'eau de la viande de porc. Liaison avec le pH. Ann. Zootech., 12, 125-1 $3^{2}$.

Goutefongea R., 1963 . Les viandes exsudatives. Ann. Zootech., 12, 297-337.

GraU R., HAMm R., I952. Eine Einfache Methode zur Bestimmung der Wasserbindung in Fleisch. Fleischwirtschaft. 4, 295.

Swift C. E., Berman M. D., I959. Factors affecting the water retention of beef. Food Technol., 15, 79-87.

Wierbicki E., Kunkle L. E., Deatherage F. E., 1957. Changes in the water holding capacity and cationic shifts during the heating and freezing and thawing of meat revealed by a simple centrifugal method for measuring shrinkage. Food Technol., 11, 69-73. 